\title{
Exposure to Mosquito Repellents Causes Profound Development Defects and Induces Oxidative Stress in Zebrafish
}

\author{
Aprathi Prabhakara ${ }^{1}$ Dechamma Pandyanda Nanjappa ${ }^{1}$ Nishith Babu ${ }^{1} \quad$ Krithika Kalladka ${ }^{1}$ \\ Anirban Chakraborty ${ }^{1}$ Gunimala Chakraborty ${ }^{1}$
}

1Division of Molecular Genetics and Cancer, Nitte University Centre for Science and Education, Nitte (Deemed to be University),

Mangalore, Karnataka, India

\begin{abstract}
Address for correspondence Gunimala Chakraborty, PhD, Division of Molecular Genetics and Cancer, Nitte University Centre for Science and Education, Nitte (Deemed to be University), Deralakatte 575018, Mangalore, Karnataka, India (e-mail: gunimala@nitte.edu.in).
\end{abstract}

J Health Allied Sci ${ }^{\mathrm{NU}}: 2020 ; 10: 122-127$

\begin{abstract}
Keywords

- zebrafish

- transfluthrin

- reactive oxygen species

- mosquito repellent

Objective The study was designed to investigate the effects of commercially available mosquito repellents on embryonic development of zebrafish.

Materials and Methods Transfluthrin is a type I pyrethroid present in all commercial mosquito and insect repellents. Pyrethrins are neurotoxins that target the nervous system of insects. Three popular brands of liquid vaporizer repellents coded as compound $A, B$, and $C$ that contained transfluthrin, ranging from 0.88 to $1.6 \% \mathrm{w} / \mathrm{w}$, were used in this study. The effects of these compounds on the embryonic development of zebrafish were investigated. In addition, the ability of transfluthrin to induce oxidative stress was examined by analyzing the generation of reactive oxygen species in exposed embryos.

Results The exposure to mosquito repellents resulted in extensive morphological defects in zebrafish embryos. The severity of the anomalies correlated with the concentration of transfluthrin in the repellents. Exposure to pure transfluthrin generated high levels of reactive oxygen species in zebrafish embryos, suggesting the induction of oxidative stress.

Conclusion Liquid vaporizer repellents are generally used for control of mosquitos and are common in many households. This study demonstrated that its exposure to mosquito repellents causes severe morphological defects and embryonic lethality in zebrafish. The study also showed that transfluthrin, the active insecticide in these repellents, induces oxidative stress in zebrafish.
\end{abstract}

\section{Introduction}

Control of mosquitos using commercially available electric repellent vaporizers is prevalent in many households around the world. ${ }^{1}$ India is endemic to several mosquito-related illnesses and the use of liquid repellents is quite common. The majority of the mosquito repellents contain active synthetic pyrethroids such as transfluthrin, allethrin, and resmethrin, which are known neurotoxins. ${ }^{2}$ The repellents are available in different forms, such as synthetic liquid, creams, and natural oils. The pyrethroids are synthetic analogues of pyrethrins, class of organic compounds normally derived from Chrysanthemum cinerariifolium that act by targeting the nervous systems of insects by blocking sodium channels hence affecting nerve conduction. ${ }^{3}$ The toxicity of pyrethroids is 2,500 times higher in insects than mammals. ${ }^{4}$ Transfluthrin, a type I pyrethroid, is a fast-acting pyrethroid insecticide with low persistence and is used very effectively against flies, mosquitoes, moths, and cockroaches in indoor environments. It is a relatively volatile substance which acts as
DOI https://doi.org/

$10.1055 / \mathrm{s}-0040-1716450$

ISSN 2582-4287.
Copyright $@ 2020$ Nitte University

(Deemed to be University)
License terms

(1) (1) $\odot \circledast$ 
an inhalation agent. Accidental inhalation and skin contacts may cause symptoms of poisoning including nervousness, anxiety, tremor, convulsions, skin allergies, sneezing, running nose, and irritation in adults. ${ }^{5}$ The compound upon oral ingestion, shows high accumulation in liver and kidney. According to World Health Organization (WHO), transfluthrins are considered as substances of low toxicity and there are no report on adverse health effect and acute toxicity of this compound. ${ }^{7}$ However, several studies on animals have shown potential detrimental effects on neurodevelopment, reproduction, and immune system after exposure to some pyrethroids. ${ }^{8}$ Accidental ingestion of these repellents by infants and small children may result in severe health issues. Long-term effects of daily transfluthrin exposure are not clear yet but there have been rising concerns over the risk of adverse toxic effects, especially in young children. Thus, investigating the effects of these compounds during early development using a suitable model system is very relevant.

In recent years, zebrafish (Danio rerio) has garnered considerable attention as an effective vertebrate model system with applications in diverse areas of biomedical research. Zebrafish scores over other model systems by virtue of its favorable qualities such as high fecundity, short generation time, embryonic transparency, ex vivo development, and low set up cost. One of the most common applications of zebrafish is in the field of toxicology to analyze the effects of drugs, environmental pollutants, and toxins. Zebrafish are small, inexpensive to maintain, and produce a large number of eggs in a single spawn, thus allowing for screening a wide range of chemicals in a relatively short time frame with high statistical significance. The larva can survive without external food for 7 days in a single well of 96 or 48 well microtiter plates. Embryos can be easily treated by waterborne exposures as they readily absorb compounds from water. They are also tolerant of dimethyl sulfoxide, which allows screening of water-insoluble compounds as well. Besides, the transparency of the larva allows in vivo assessment of effects including visual observation of important organs. Thus, the model system combines the scalability of the in vitro system with the ability to perform the whole animal-based phenotypic assays. $^{8}$

Here, we describe the developmental defects associated with exposure of zebrafish embryos to commercially available liquid repellents that contain transfluthrin as the active insecticide. Our data also reveal that transfluthrin exposure generates ROS in zebrafish and causes oxidative stress.

\section{Materials and Methods}

\section{Zebrafish Husbandry and Rearing}

Adult zebrafish maintained at rearing facility with temperature $28 \pm 0.5^{\circ} \mathrm{C}$ and $14 / 10$ hours light and day dark cycle were used in the study. Pairwise mating of fishes was done to collect eggs. The fertilized eggs were collected the next morning, transferred into petri dishes containing embryonic (E3) medium and were maintained at $28^{\circ} \mathrm{C}$ in a cooling incubator. The untreated/unexposed embryos served as control.

\section{Types of Liquid Repellents}

Three most popular commercially available liquid mosquito repellents were selected for the study and were coded as A, B, and $C$ based on the concentration of transfluthrin. The repellents were categorized into three types; one with high concentration of transfluthrin (code A, 1.6\%), one with medium concentration of transfluthrin (code B, 1.22\%), and one with low concentration of transfluthrin (code C, $0.88 \%$ ).

\section{Mode of Exposure}

A semi-static methodology was followed where growth medium change and the renewal of the test solution were done twice a day. The embryos were exposed at 10 hours post fertilization (hpf) and the effects on development were monitored at $24,48,72$, and $120 \mathrm{hpf}$, respectively. The experiments were conducted in 12-well microtiter plate, with 8-10 embryos in each well. Since the repellents used in the study were commercially available liquid vaporizers, the exposure to the compounds was done in percentage of total volume of embryonic water (E3 medium). A range of concentrations of repellents starting from 0.1 to $20 \%$ of the total volume of water was tested. Pure transfluthrin (99.2\%, Sigma) was used in ppm levels.

\section{Evaluation of Developmental Toxicity}

The developmental toxicity was evaluated for each concentration through visual observation and recording of a set of lethal and sublethal end points over a period of 5 days with a change of medium and renewal of test solution twice a day. The experiments were conducted in triplicates with 8 to 10 embryos in each replicate. Images from three replicates for each end point for each concentration were recorded using a stereomicroscope (Leica S9D, Germany) and were compared with the control (unexposed) embryos. The teratogenic effects were recorded on day 1 (24 hpf), whereas the cardiotoxicity evaluation was done on day 2 ( $48 \mathrm{hpf}$ ). The effects on hatching and swimming behavior were evaluated on day 3 (72 hpf). The difference in the number of surviving embryos at each day was statistically compared with that of control. The $p$-value was calculated using a $t$-test, and a value of $<0.05$ was considered statistically significant.

\section{Evaluation of ROS Activity}

The generation of ROS was checked by the addition of 2', 7'dichlorodihydrofluorescein-diacetate ( $\left.\mathrm{H}_{2} \mathrm{DCFDA}\right)$ (Sigma, United States), a cell permeant, that acts as an indicator of ROS. During oxidation, the acetate groups are cleaved and the release of esterases converts a nonfluorescent $\mathrm{H}_{2}$ DCFDA into a detectable fluorescent form. Pure transfluthrin was used to check the generation of ROS. Transfluthrin was exposed at $10 \mathrm{hpf}$ and the evaluation of ROS was performed at 24 and 48 hpf. Embryos treated with $\mathrm{H}_{2} \mathrm{O}_{2}$ were used as positive control. Briefly, transfluthrin-treated embryos ${ }^{5-10}$ were transferred into a new 24-well plate and washed with PBS. The washed embryos were incubated in $5-\mu \mathrm{M} \mathrm{H}_{2} \mathrm{DCFDA}$ for 2 hours. Post incubation, the embryos were washed and observed under a fluorescent microscope (Lieca DM2500, Germany). 


\section{Results}

\section{Effects of Mosquito Repellents on Zebrafish Embryonic Development}

The assays for developmental toxicities revealed that all the three repellents were highly toxic even at concentrations ranging from 0.1 to $0.5 \%$. The observations were made by counting the embryos at each day. The embryos were categorized into three groups: alive without defects, alive with defects, and dead. The absence of the heart beat was considered as an indication of mortality. The summary of the observations is shown in - Tables $\mathbf{1}$ to $\mathbf{3}$. There was no significant death or morphological abnormalities at lower concentrations $(0.1-0.5 \%)$ for any of the repellents on day 1 and day 2 (-Figs. 1A, 2A, and 3A). However, at moderate (1 and $5 \%$ ) and higher concentrations (10 and $20 \%$ ), the morphological abnormalities and lethality of embryos were evident for all the three repellents from day 1 . In addition, the difference in the number of surviving embryos (with or without morphological defects) in treated and control was statistically significant $(p<0.05)$ for all the repellents and the reduction in the number of embryos in treated conditions was directly proportional to the concentrations of the repellents used. In case of repellents A and B, which contained high and moderate concentration of transfluthrin, respectively (1.6 and $1.22 \%$ ), the majority of the embryos showed lethality only on day 5 at lower concentrations (0.1-0.5\%) without any significant difference in the number of surviving embryos at earlier time points ( $\mathbf{- F i g s . ~ 1 A , ~ 2 A , ~ a n d ~} \mathbf{3 A}$ ). In contrast, in case of repellent $C$, which contained lower concentration of transfluthrin $(0.88 \%)$, the majority of the embryos were alive with or without morphological abnormalities (-Figs. 1A, 2A, and 3A) with $0.3 \%$ showing a significant difference between the treated and the control. Thus, the effects on morphological development were directly proportional to the concentration of transfluthrin in the repellents with repellent $A$ being the most toxic and repellent $C$ being the least toxic. At moderate concentrations (1-5\%), exposure to repellent $A$ and $B$ resulted in significantly less number of surviving embryos at day 2 and day 3 with 100\% lethality by day 3 ( - Figs. 1A, 2A). In case of repellent $C$, despite a significant difference in number of surviving embryos at day 2 and day $3(\boldsymbol{- F i g}$. $\mathbf{3 A})$, complete mortality was seen only at

Table 1 The summary of morphological observations of zebrafish embryos across $5 \mathrm{~d}$ of development after exposure to repellent $\mathrm{A}$ (transfluthrin: 1.6\%)

\begin{tabular}{|c|c|c|c|c|c|c|c|c|c|c|c|c|}
\hline \multirow{2}{*}{$\begin{array}{l}\text { Concentration } \\
\text { of repellent } \\
\text { (\% of embryo } \\
\text { water) } N=30\end{array}$} & \multicolumn{3}{|c|}{ Day 1} & \multicolumn{3}{|c|}{ Day 2} & \multicolumn{3}{|c|}{ Day 3} & \multicolumn{3}{|c|}{ Day 5} \\
\hline & Dead & $\begin{array}{l}\text { Alive } \\
\text { without } \\
\text { defects }\end{array}$ & $\begin{array}{l}\text { Alive } \\
\text { with } \\
\text { defects }\end{array}$ & Dead & $\begin{array}{l}\text { Alive } \\
\text { without } \\
\text { defects }\end{array}$ & $\begin{array}{l}\text { Alive } \\
\text { with } \\
\text { defects }\end{array}$ & Dead & $\begin{array}{l}\text { Alive } \\
\text { without } \\
\text { defects }\end{array}$ & $\begin{array}{l}\text { Alive } \\
\text { with } \\
\text { defects }\end{array}$ & Dead & $\begin{array}{l}\text { Alive } \\
\text { without } \\
\text { defects }\end{array}$ & $\begin{array}{l}\text { Alive } \\
\text { with } \\
\text { defects }\end{array}$ \\
\hline 0.1 & 0 & 30 & 0 & 2 & 28 & 0 & 1 & 4 & 23 & 27 & 0 & 0 \\
\hline 0.2 & 0 & 30 & 0 & 0 & 30 & 0 & 2 & 0 & 28 & 28 & 0 & 0 \\
\hline 0.32 & 0 & 30 & 0 & 2 & 28 & 0 & 0 & 8 & 20 & 28 & 0 & 0 \\
\hline 0.5 & 0 & 30 & 0 & 1 & 29 & 0 & 0 & 0 & 29 & 29 & 0 & 0 \\
\hline 1.0 & 0 & 30 & 0 & 0 & 30 & 0 & 20 & 0 & 10 & 10 & 0 & 0 \\
\hline 5.0 & 2 & 0 & 28 & 1 & 0 & 27 & 27 & 0 & 0 & - & - & - \\
\hline 10.0 & 9 & 0 & 21 & 20 & 0 & 1 & 1 & 0 & 0 & - & - & - \\
\hline 20.0 & 4 & 0 & 26 & 22 & & 4 & 4 & 0 & 0 & - & - & - \\
\hline
\end{tabular}

Table 2 The summary of morphological observations of zebrafish embryos across $5 \mathrm{~d}$ of development after exposure to repellent B (transfluthrin:1.22\%)

\begin{tabular}{|c|c|c|c|c|c|c|c|c|c|c|c|c|}
\hline \multirow{2}{*}{$\begin{array}{l}\text { Concentration of } \\
\text { repellent (\% of } \\
\text { embryo water) } \\
N=30\end{array}$} & \multicolumn{3}{|c|}{ Day 1} & \multicolumn{3}{|c|}{ Day 2} & \multicolumn{3}{|c|}{ Day 3} & \multicolumn{3}{|c|}{ Day 5} \\
\hline & Dead & $\begin{array}{l}\text { Alive } \\
\text { without } \\
\text { defects }\end{array}$ & $\begin{array}{l}\text { Alive } \\
\text { with } \\
\text { defects }\end{array}$ & Dead & $\begin{array}{l}\text { Alive } \\
\text { without } \\
\text { defects }\end{array}$ & $\begin{array}{l}\text { Alive } \\
\text { with } \\
\text { defects }\end{array}$ & Dead & $\begin{array}{l}\text { Alive } \\
\text { without } \\
\text { defects }\end{array}$ & $\begin{array}{l}\text { Alive } \\
\text { with } \\
\text { defects }\end{array}$ & Dead & $\begin{array}{l}\text { Alive } \\
\text { without } \\
\text { defects }\end{array}$ & $\begin{array}{l}\text { Alive with } \\
\text { defects }\end{array}$ \\
\hline 0.1 & 0 & 30 & 0 & 0 & 30 & 0 & 10 & 10 & 10 & 10 & 0 & 10 \\
\hline 0.2 & 0 & 30 & 0 & 0 & 30 & 0 & 2 & 10 & 18 & 10 & 0 & 10 \\
\hline 0.3 & 0 & 30 & 0 & 0 & 30 & 0 & 1 & 0 & 29 & 28 & 0 & 1 \\
\hline 0.35 & 0 & 30 & 0 & 2 & 28 & 0 & 0 & 8 & 20 & 28 & 0 & 0 \\
\hline 0.5 & 0 & 30 & 0 & 0 & 3 & 27 & 1 & 0 & 29 & 29 & 0 & 0 \\
\hline 1.0 & 1 & 29 & 0 & 5 & 0 & 24 & 24 & 0 & 0 & - & - & - \\
\hline 5.0 & 2 & 0 & 28 & 3 & 0 & 25 & 25 & 0 & 0 & - & - & - \\
\hline 10.0 & 0 & 0 & 30 & 1 & 0 & 29 & 29 & 0 & 0 & - & - & - \\
\hline 20.0 & 0 & 0 & 30 & 22 & & 8 & 8 & 0 & 0 & - & - & - \\
\hline
\end{tabular}


Table 3 The summary of morphological observations of zebrafish embryos across $5 \mathrm{~d}$ of development after exposure to repellent $\mathrm{C}$ (transfluthrin: 0.88\%)

\begin{tabular}{|c|c|c|c|c|c|c|c|c|c|c|c|c|}
\hline \multirow{2}{*}{$\begin{array}{l}\text { Concentration } \\
\text { of repellent } \\
\text { (\% of embryo } \\
\text { water), } N=20\end{array}$} & \multicolumn{3}{|c|}{ Day 1} & \multicolumn{3}{|c|}{ Day 2} & \multicolumn{3}{|c|}{ Day 3} & \multicolumn{3}{|c|}{ Day 5} \\
\hline & Dead & $\begin{array}{l}\text { Alive } \\
\text { without } \\
\text { defects }\end{array}$ & $\begin{array}{l}\text { Alive } \\
\text { with } \\
\text { defects }\end{array}$ & Dead & $\begin{array}{l}\text { Alive } \\
\text { without } \\
\text { defects }\end{array}$ & $\begin{array}{l}\text { Alive } \\
\text { with } \\
\text { defects }\end{array}$ & Dead & $\begin{array}{l}\text { Alive } \\
\text { without } \\
\text { defects }\end{array}$ & $\begin{array}{l}\text { Alive } \\
\text { with } \\
\text { defects }\end{array}$ & Dead & $\begin{array}{l}\text { Alive } \\
\text { without } \\
\text { defects }\end{array}$ & $\begin{array}{l}\text { Alive } \\
\text { with } \\
\text { defects }\end{array}$ \\
\hline 0.1 & 0 & 20 & 0 & 0 & 20 & 0 & 0 & 20 & 0 & 1 & 17 & 2 \\
\hline 0.2 & 0 & 20 & 0 & 0 & 20 & 0 & 1 & 19 & 0 & 1 & 18 & 0 \\
\hline 0.3 & 0 & 20 & 0 & 2 & 18 & 0 & 1 & 17 & 0 & 5 & 12 & 0 \\
\hline 0.4 & 0 & 20 & 0 & 1 & 19 & 0 & 0 & 10 & 9 & 4 & 0 & 15 \\
\hline 0.5 & 2 & 18 & 0 & 0 & 18 & 0 & 0 & 0 & 18 & 18 & 0 & 0 \\
\hline 1.0 & 0 & 20 & 0 & 0 & 20 & 0 & 4 & 0 & 16 & 16 & - & - \\
\hline 5.0 & 0 & 20 & 0 & 0 & 0 & 20 & 5 & 0 & 15 & 15 & - & - \\
\hline 10.0 & 7 & 0 & 13 & 13 & 0 & 0 & - & - & - & - & - & - \\
\hline 20.0 & 20 & 0 & 0 & - & - & - & - & - & - & - & - & - \\
\hline
\end{tabular}
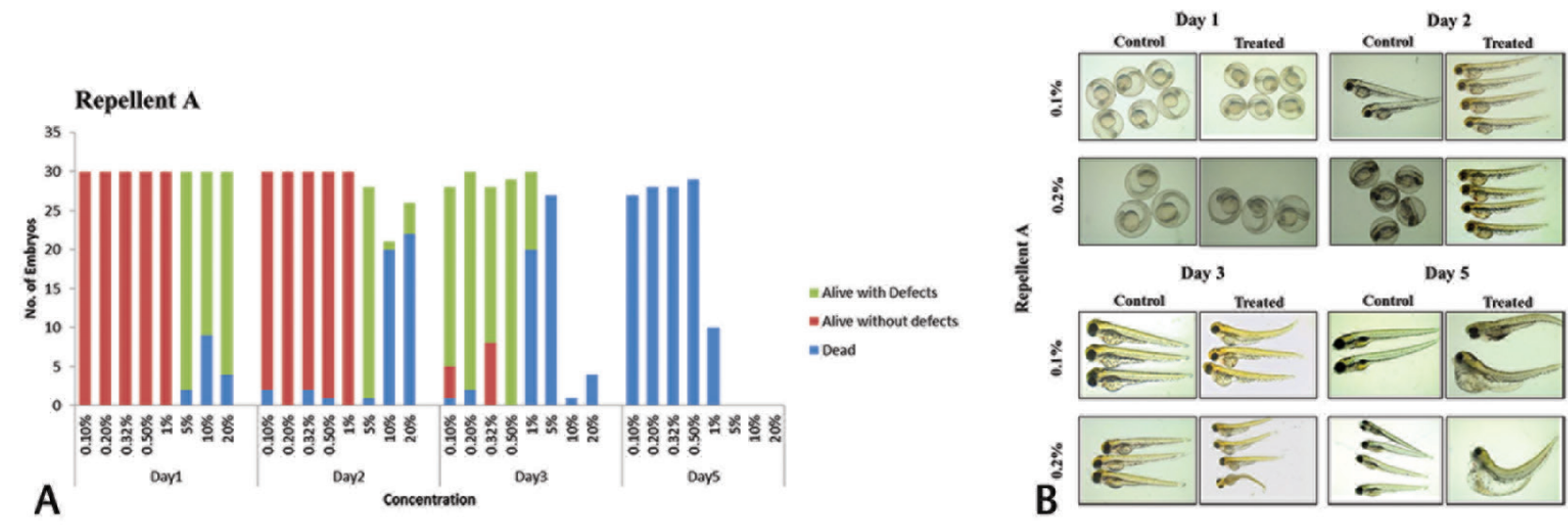

Fig. 1 (A) Survival rates of zebrafish embryos treated with different concentrations of repellent A. The embryos were exposed at 10-hour post-fertilization (hpf) and the total number of embryos that survived with or without associated developmental defects and that died were recorded at $24 \mathrm{hpf}$ (day 1), 48 (day 2), 72 (day 3), and $120 \mathrm{hpf}$ (day 5). “*” indicates $p<0.05$. (B) Representative images of zebrafish embryos at different stages of development after exposure to 0.1 and $0.2 \%$ of repellent A. Morphological anomalies such as pericardial edema, incomplete yolk absorption (roundish yolk), and upward body curvature are evident in treated embryos at day 5.
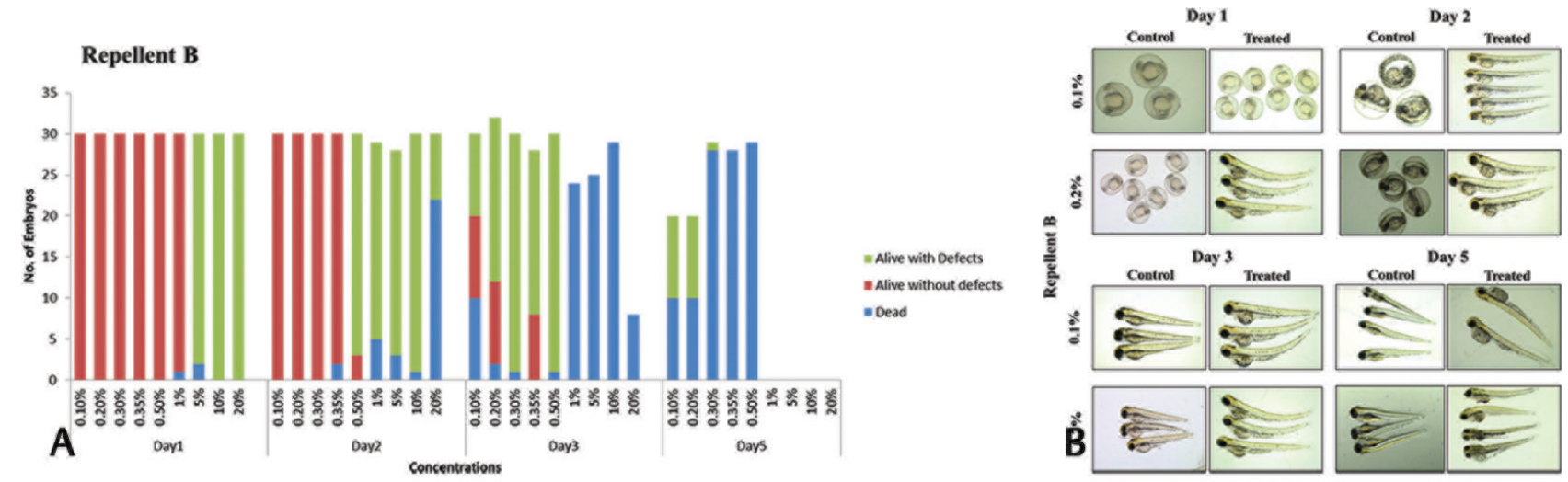

Fig. 2 (A) Survival rates of zebrafish embryos treated with different concentrations of repellent B. The embryos were exposed at 10-hour post-fertilization (hpf) and the total number of embryos that survived with or without associated developmental defects and that died were recorded at $24 \mathrm{hpf}$ (day 1), 48 (day 2), 72 (day 3), and 120 hpf (day 5). "*” indicates $p<0.05$. (B) Representative images of zebrafish embryos at different stages of development after exposure to 0.1 and $0.2 \%$ of repellent B. Morphological anomalies such as incomplete yolk absorption (roundish yolk) and mild pericardial edema are evident in treated embryos at day 3 and day 5 . 

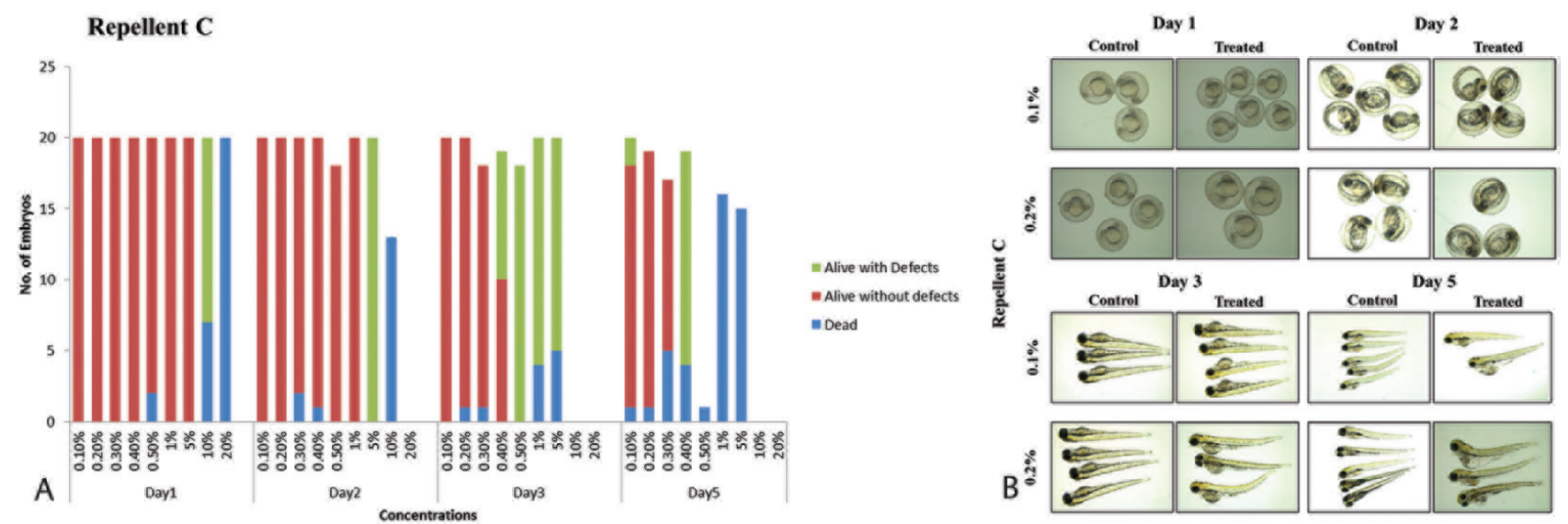

Fig. 3 (A) Survival rates of zebrafish embryos treated with different concentrations of repellent C. The embryos were exposed at 10-hour post-fertilization (hpf) and the total number of embryos that survived with or without associated developmental defects and that died were recorded at $24 \mathrm{hpf}$ (day 1), 48 (day 2), 72 (day 3), and $120 \mathrm{hpf}$ (day 5). “*” indicates $p<0.05$. (B) Representative images of zebrafish embryos at different stages of development after exposure to 0.1 and $0.2 \%$ of repellent C. Morphological anomalies such as incomplete yolk absorption (roundish yolk), lack of digestive track, and smaller body are evident in treated embryos at day 5 .

day 5 ( - Fig. 3A), further suggesting a dose-dependent effect of transfluthrin. At higher concentrations (10 and 20\%) all the three repellents were highly toxic with complete mortality by day 2 and 3 . Even at day 1 , there were significantly less number of surviving embryos in case repellent $A$ and $C$ (-Figs. 1A and 3A). The morphological abnormalities associated with repellent exposure included pericardial edema, incomplete yolk absorption, upward body curvature, and absence of gut-associated organs. The representative images are shown in - Figs. 1B, 2B, and 3B.

\section{Induction of Oxidative Stress by Transfluthrin}

Pyrethroids are known to be capable of generating free radicals and causing stress on cells. To investigate whether exposure to transfluthrin generates reactive oxygen species (ROS) in zebrafish embryos, $\mathrm{H}_{2}$ DCFDA staining was performed. Oxidative stress is a result of imbalance between free radical generation and antioxidant production. Increased generation of ROS is a hallmark of oxidative stress. The results of $\mathrm{H}_{2}$ DCFDA staining indicated that transfluthrin exposure leads to the generation of high levels of ROS, suggesting the induction of oxidative stress. Representative images are shown in - Fig. 4.

\section{Discussion}

Transfluthrin, a synthetic type I pyrethroid, is the most commonly used insecticide in mosquito repellents. Mosquito repellents are marketed in various forms such as creams, coils, one-push aerosols, and in liquid form. The liquid repellents, which work as vaporizers, are the most preferred one in households around the world. The actual concentration of transfluthrin in the liquid repellents varies and it generally ranges from 1.6 to $0.6 \%$. Although pyrethroids are considered nontoxic for mammals, there have been reports of pyrethroid poisoning, particularly transfluthrin poisoning in human. ${ }^{10}$ The major symptoms of the transfluthrin poisoning in adults include headache,

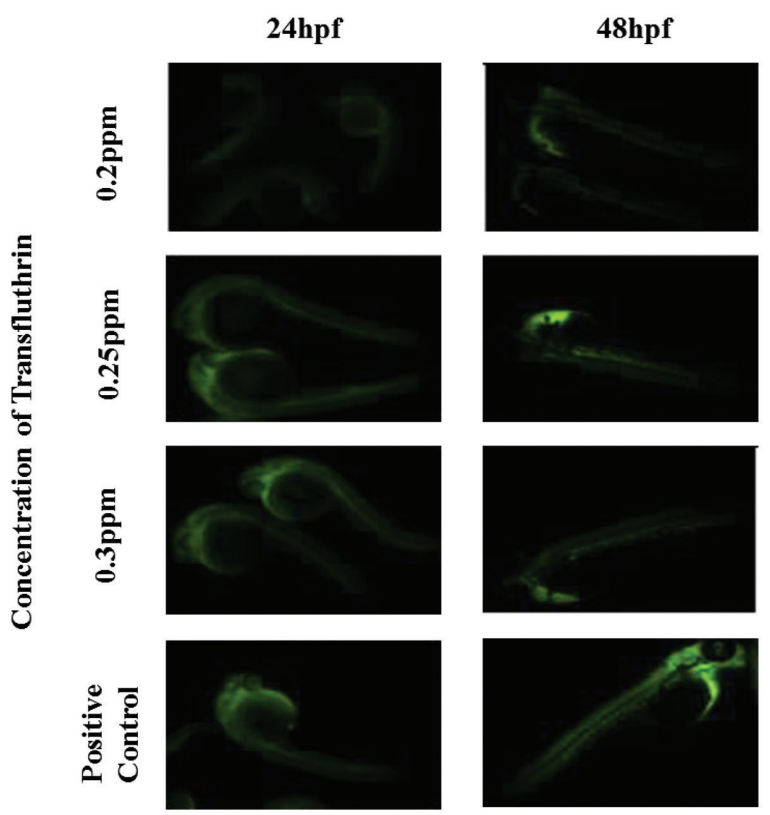

Fig. 4 Detection of ROS in zebrafish embryos treated with pure transfluthrin. Representative images of $\mathrm{H}_{2}$ DCFDA staining in zebrafish embryos at 24 and 48 hpf after exposure to different concentrations of transfluthrin. Zebrafish embryos exposed to $\mathrm{H}_{2} \mathrm{O}_{2}$ served as positive control. $\mathrm{H}_{2}$ DCFDA, 2', 7'-dichlorodihydrofluoresceindiacetate; ROS, reactive oxygen species.

dizziness, drowsiness, epigastric pain, vomiting, anorexia, fatigue, muscle twitching, salivation, running nose, and convulsions. ${ }^{11}$

In this study, the toxicities of three commercially available mosquito repellents were analyzed in zebrafish model system. The results clearly indicated that transfluthrin is extremely toxic to zebrafish embryos and even low concentrations of 0.1 to $0.5 \%$, it interfered with the survivability of the embryos. The results further revealed that the deformities and the lethality associated with transfluthrin were dose-dependent with repellents containing higher 
concentrations of transfluthrin (1.6\%) being the most toxic compared with the type that contained lower concentration of transfluthrin $(0.88 \%)$. Our data corroborated with an earlier study, which reported that the mortality of the zebrafish embryo increased with the increase in the concentration and exposure time of cypermethrin, a synthetic pyrethroid. ${ }^{12}$ Our data also demonstrated that transfluthrin induces the generation of ROS in zebrafish embryos. Cypermethrin and fenvalerate, which belong to the pyrethroid family, have been shown to produce ROS in cotton bollworm..$^{13}$ Thus, our results are in accordance with previously published reports.

Accidental ingestion of liquid mosquito repellents can result in transfluthrin poisoning which manifests as intravascular hemolysis and methemoglobinemia, uncontrolled seizures, and rise in leucocytes counts in juveniles and adults. ${ }^{11}$ A study in mice has shown that chronic exposure to transfluthrin (20-25\%) over a period of 15 days can lead to substantial damage to organs such as lung, liver, and kidney. ${ }^{14}$

Although the liquid repellents used in households are vaporizers, their extensive use makes them accessible to infants and children who might ingest them accidentally or inadvertently leading to disastrous consequences. The focus of this study was to analyze the effects of acute exposure of mosquito liquid repellents on zebrafish embryonic development. Our data clearly indicated that these repellents are highly toxic to zebrafish at early stages of development. Even at very low concentrations, these repellents caused severe morphological deformities and mortality. In addition, the toxicity depended on the concentration of transfluthrin, the active compound, in these repellents. Further studies would be required to understand the mechanisms of action and to decode the pathways involved in transfluthrin poisoning.

\section{Funding}

The financial support from Nitte (Deemed to be University) through the intramural research grant (NUSR2/2018/10/50) to the first and the fifth author is gratefully acknowledged.

\section{Conflict of Interest}

None declared.

\section{References}

1 van Eijk AM, Ramanathapuram L, Sutton PL, et al. The use of mosquito repellents at three sites in India with declining malaria transmission: surveys in the community and clinic. Parasit Vectors 2016;9(1):418

2 Shafer TJ, Meyer DA, Crofton KM. Developmental neurotoxicity of pyrethroid insecticides: critical review and future research needs. Environ Health Perspect 2005;113(2):123-136

3 Gajendiran A, Abraham J. An overview of pyrethroid insecticides. Front Biol 2018;13:79-90

4 Bradberry SM, Cage SA, Proudfoot AT, Vale JA. Poisoning due to pyrethroids. Toxicol Rev 2005;24(2):93-106

5 Pauluhn J, Ozaki K. Transfluthrin: comparative efficacy and toxicity of reference and generic versions. Regul Toxicol Pharmacol 2015;71(1):78-92

6 Chandelia S, Dubey NK. Mosquito repellent vaporizer poisoning-is the culprit transfluthrin or kerosene.? Indian Pediatr 2014;51(4):319-320

7 Feo ML, Eljarrat E, Barceló D. Determination of pyrethroid insecticides in environmental samples. Trends Analyt Chem 2010;29:692-705

8 ATSDR, Agency for Toxic Substances and Disease Registry. Clement International Corp. 2000. Available at: https://www. atsdr.cdc.gov/. Accessed August 20, 2020

9 Yang L, Ho NY, Alshut R, et al. Zebrafish embryos as models for embryotoxic and teratological effects of chemicals. Reprod Toxicol 2009;28(2):245-253

10 Westerfield M. The Zebrafish Book: A Guide for the Laboratory Use of Zebrafish (Brachydanio rerio). Corevallis, OR: University of Oregon Press; 1995

11 Sachdev A, Gulla KM, Anand K, Raheja K, Gupta N. Transfluthrin poisoning resulting in intra vascular haemolysis and methemoglobinemia in G6PD deficiency-treatment challenge. J Clin Toxicol 2015;5:2

12 Sathya K, Thiripurasundari M, Uma A, Srinivasan MR. Subas Chandra Bose M. The toxic effect of cypermethrin on zebrafish embryo-an alternative model for assessing the embryo toxicity of environmental chemicals. Indo Am J Pharm Res 2014;4:2316-2322

13 Akbar SM, Sharma HC, Jayalakshmi SK, Sreeramulu K. Effect of pyrethroids, permethrin and fenvalarate, on the oxidative stress of Helicoverpa armigera. World J Sci Technol 2012;2:1-5

14 Juswono UP, Wardoyo AY, Widodo CS, Andoyo J, Noor E. The effects of transfluthrin as the active substance of one push aerosol repellent on organs damage of mice (mus musculus) (case study of lung, liver, bloods, and kidney) Int J 2019; $16: 113-118$ 